\title{
Angular analysis of $B_{d}^{0} \rightarrow K^{*} \mu^{+} \mu^{-}$decay with the ATLAS detector
}

\section{Ina Carli*}

Charles University

E-mail: Ina.Carli@cern.ch

\begin{abstract}
An angular analysis of the decay $B_{d}^{0} \rightarrow K^{*} \mu^{+} \mu^{-}$is presented using LHC data from proton-proton collisions at $\sqrt{s}=8 \mathrm{TeV}$ collected with the ATLAS detector in 2012. The study is based on 20.3 $\mathrm{fb}^{-1}$ of integrated luminosity. Measurements of the $K^{*}$ longitudinal polarisation fraction and a set of angular parameters obtained for this decay are presented. The results are consistent with Standard Model predictions.
\end{abstract}

The 15th International Conference on Flavor Physics \& CP Violation 5-9 June 2017

Prague, Czech Republic

* Speaker.

${ }^{\dagger}$ On behalf of the ATLAS collaboration. 


\section{1. $B_{d}^{0} \rightarrow K^{*} \mu^{+} \mu^{-}$decay in the Standard Model}

The production of $b$-hadrons at the Large Hadron Collider (LHC) [1] provides excellent opportunities for indirect searches for new physics. The existing studies often include rare decays of $b$-hadrons mediated by flavour-changing neutral currents (FCNC) which are forbidden at tree level in the Standard Model (SM). They occur at higher orders as loop diagrams and therefore are sensitive to contributions of physics beyond SM such as heavy particles in the loops.

The decay $B_{d}^{0} \rightarrow K^{*}(892) \mu^{+} \mu^{-}$is an example of an FCNC process with $b \rightarrow s \mu^{+} \mu^{-}$transition at the quark level. The lowest order Feynman diagrams of this process in SM are shown in Figure 1. Angular distributions of the decay products, together with differential branching fraction, isospin asymmetry, and ratio of rates of the decay into dimuon and dielectron final states are all potentially sensitive to different types of new physics. We present recent results of an angular analysis of $B_{d}^{0} \rightarrow K^{*}(892) \mu^{+} \mu^{-}$decay performed by the ATLAS Collaboration [2]. Hereafter, the $K^{*}(892)$ is referred to as $K^{*}, B_{d}^{0}$ as $B^{0}$ and charge conjugation is implied throughout, unless stated otherwise.
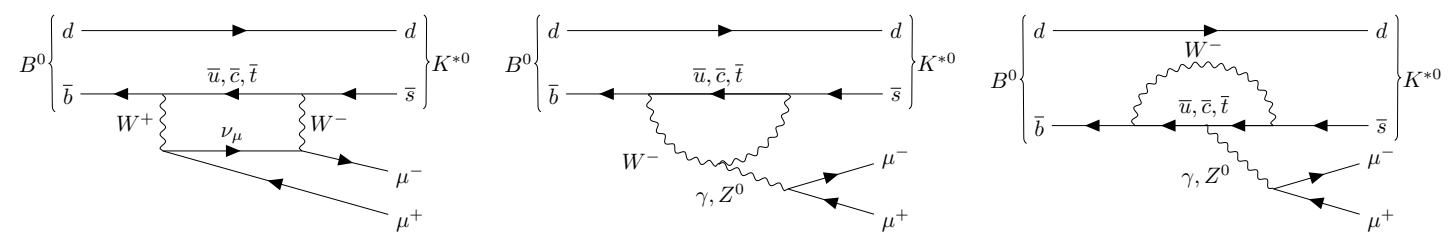

Figure 1: Box and penguin Feynman diagrams of the $B^{0} \rightarrow K^{*} \mu^{+} \mu^{-}$decay in the SM.

The kinematics of $B^{0} \rightarrow K^{*} \mu^{+} \mu^{-}$decay with subsequent $K^{*} \rightarrow K^{+} \pi^{-}$process can be described by four variables: the invariant mass $q^{2}$ of the dimuon system and three angles defining configuration of the final state. Different conventions for angular observables are adopted in literature, this analysis uses a definition described by the LHCb Collaboration in Ref. [3] and illustrated in Figure 2: $\theta_{K}$ is the angle between the $K^{+}$and the direction opposite to the $B^{0}$ in the $K^{*}$ rest frame, $\theta_{L}$ is the angle between the $\mu^{+}$and the direction opposite to the $B^{0}$ in the dimuon rest frame and $\phi$ the angle between the two decay planes formed by the $K \pi$ and the dimuon systems in the $B^{0}$ rest frame. For $\overline{B^{0}}$ mesons the definitions are given with respect to the negatively charged particles.

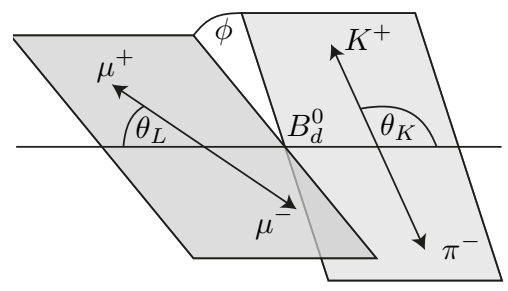

Figure 2: Definition of angular observables of the $B^{0} \rightarrow K^{*} \mu^{+} \mu^{-}$decay: angles $\theta_{K}, \theta_{L}$ and $\phi$ are measured in the rest frames of $K^{*}$, dimuon system and $B^{0}$, respectively.

The differential decay rate of $B^{0} \rightarrow K^{*} \mu^{+} \mu^{-}$can be expressed as a function of angular coef- 
ficients $S_{i}$ and fraction $F_{\mathrm{L}}$ of longitudinally polarised $K^{*}$ mesons as

$$
\begin{aligned}
\frac{1}{\mathrm{~d} \Gamma / \mathrm{d} q^{2}} & \frac{\mathrm{d}^{4} \Gamma}{\mathrm{d} \cos \theta_{L} \mathrm{~d} \cos \theta_{K} \mathrm{~d} \phi \mathrm{d} q^{2}}=\frac{9}{32 \pi}\left[\frac{3\left(1-F_{\mathrm{L}}\right)}{4} \sin ^{2} \theta_{K}+F_{\mathrm{L}} \cos ^{2} \theta_{K}+\frac{1-F_{\mathrm{L}}}{4} \sin ^{2} \theta_{K} \cos 2 \theta_{L}\right. \\
& -F_{\mathrm{L}} \cos ^{2} \theta_{K} \cos 2 \theta_{L}+S_{3} \sin ^{2} \theta_{K} \sin ^{2} \theta_{L} \cos 2 \phi+S_{4} \sin 2 \theta_{K} \sin 2 \theta_{L} \cos \phi \\
& +S_{5} \sin 2 \theta_{K} \sin \theta_{L} \cos \phi+S_{6} \sin ^{2} \theta_{K} \cos \theta_{L}+S_{7} \sin 2 \theta_{K} \sin \theta_{L} \sin \phi \\
& \left.+S_{8} \sin 2 \theta_{K} \sin 2 \theta_{L} \sin \phi+S_{9} \sin ^{2} \theta_{K} \sin ^{2} \theta_{L} \sin 2 \phi\right]
\end{aligned}
$$

All coefficients can be extracted from a fit to this function. However, this requires a minimum signal yield and purity in order to avoid pathological fit behaviour. This issue can be mitigated using trigonometric transformations to fold angular distributions and simplify Equation (1.1).

The folding schema used here was proposed by Ref. [4] and results in four sets of transformations, such that three parameters can be extracted from each of four fits: $F_{\mathrm{L}}, S_{3}$ and one of the other $S_{i}$ parameters. As a consequence, $S_{6}$ and $S_{9}$ cannot be extracted from the data. For example, the folding schema for extracting $S_{4}$ is defined by transformations

$$
\begin{array}{ll}
\phi \rightarrow-\phi & \text { if } \phi<0, \\
\phi \rightarrow \pi-\phi, \theta_{L} \rightarrow \pi-\theta_{L} & \text { if } \theta_{L}>\pi / 2
\end{array}
$$

and results in new effective ranges of the angular variables, $\cos \theta_{L} \in[0,1], \cos \theta_{K} \in[-1,1]$ and $\phi \in$ $[0, \pi]$. The differential decay rate can then be written as a function of $F_{\mathrm{L}}, S_{3}$ and $S_{4}$ :

$$
\begin{aligned}
\frac{1}{\mathrm{~d} \Gamma / \mathrm{d} q^{2}} & \frac{\mathrm{d}^{4} \Gamma}{\mathrm{d} \cos \theta_{L} \mathrm{~d} \cos \theta_{K} \mathrm{~d} \phi \mathrm{d} q^{2}}=\frac{9}{8 \pi}\left[\frac{3\left(1-F_{\mathrm{L}}\right)}{4} \sin ^{2} \theta_{K}+F_{\mathrm{L}} \cos ^{2} \theta_{K}+\frac{1-F_{\mathrm{L}}}{4} \sin ^{2} \theta_{K} \cos 2 \theta_{L}\right. \\
& \left.-F_{\mathrm{L}} \cos ^{2} \theta_{K} \cos 2 \theta_{L}+S_{3} \sin ^{2} \theta_{K} \sin ^{2} \theta_{L} \cos 2 \phi+S_{4} \sin 2 \theta_{K} \sin 2 \theta_{L} \cos \phi\right] .
\end{aligned}
$$

The $S_{i}$ parameters are very sensitive to a choice of hadronic form factors, and thus their predictions have significant theoretical uncertainties. To reduce these uncertainties and improve the sensitivity to new physics, a set of optimised parameters $P_{i}$ was proposed in Ref. [5] as following:

$$
P_{1}=\frac{2 S_{3}}{1-F_{\mathrm{L}}}, \quad P_{2}=\frac{S_{6}}{2\left(1-F_{\mathrm{L}}\right)}, \quad P_{3}=-\frac{S_{9}}{1-F_{\mathrm{L}}}, \quad P_{i=4,5,6,8}^{\prime}=\frac{S_{j=4,5,7,8}}{\sqrt{F_{\mathrm{L}}\left(1-F_{\mathrm{L}}\right)}} .
$$

The $B^{0} \rightarrow K^{*} \mu^{+} \mu^{-}$decay is self-tagging, i.e. the flavour of the $B^{0}$ meson can be inferred from the charge of the kaon track. All angular parameters are defined such that they have the same distribution for the $\overline{B^{0}}$ sample. Therefore it is possible to extract them from fits to data containing both $B^{0}$ and $\overline{B^{0}}$ decays.

\section{Dataset and event selection}

This study uses $20.3 \mathrm{fb}^{-1}$ of $p p$ collision data at a centre-of-mass energy $\sqrt{s}=8 \mathrm{TeV}$ recorded by the ATLAS detector [6] in 2012. Results are presented in six different bins of $q^{2}$ in the range 0.04 to $6 \mathrm{GeV}^{2}$, where three of these bins overlap. 
A number of Monte Carlo (MC) simulated event samples were generated and reconstructed in the same way as data. Two samples contained signal decay simulated with angular distributions following SM predictions and were used to study signal selection, mistagging and reconstruction differences between $B^{0}$ and $\overline{B^{0}}$ decays. The third sample had $F_{\mathrm{L}}=1 / 3$ and uniform distributions of $\cos \theta_{K}, \cos \theta_{L}$ and $\phi$ to study the detector, reconstruction and selection acceptance. Several other MC samples were generated to study backgrounds potentially contributing to the signal regions.

Data were combined from 19 trigger chains with various signatures and thresholds in order to maximise the signal yield. The largest contribution came from chains requiring one muon with a transverse momentum $p_{\mathrm{T}}>4 \mathrm{GeV}$ and the other muon with $p_{\mathrm{T}}>6 \mathrm{GeV}$. The effective trigger efficiency for the offline-selected signal events was about $29 \%$.

Each signal candidate was reconstructed offline from a pair of oppositely charged muons and a pair of tracks with $|\eta|<2.5$ and $p_{\mathrm{T}}>3.5(0.5) \mathrm{GeV}$ for muon (pion and kaon) tracks. The muon pair was accepted if the vertex fit quality was $\chi^{2} / N D F<10$. The $K^{*}$ was required to have invariant mass in the range $[846,946] \mathrm{MeV}$ and $p_{\mathrm{T}}>3 \mathrm{GeV}$. Since ATLAS detector does not have a dedicated charged-particle identification subsystem, $K^{*}$ candidates were reconstructed from pairs of oppositely charged tracks with both possible $K \pi$ mass hypotheses. If both candidates satisfied all cuts, they were retained and one of them was selected in the next step.

The $B^{0}$ candidates were constructed from $K^{*}$ and dimuon candidates by fitting the four tracks in a common vertex. To suppress background, the fit quality was required to be $\chi^{2} / N D F<2$ and the $B^{0}$ lifetime significance $\tau / \sigma_{\tau}>12.5$, where the decay time uncertainty $\sigma_{\tau}$ was calculated from covariance matrices associated with fits of the secondary $B^{0}$ and primary vertices. It was required that the pointing angle $\Phi$, defined as angle between the vector from the primary vertex to the $B^{0}$ vertex and the $B^{0}$ momentum, should satisfy $\cos \Phi>0.999$. It was observed that a significant amount of background from partially reconstructed $B \rightarrow \mu^{+} \mu^{-} X$ decays accumulates in the invariant mass below the $B^{0}$ mass. This contribution was partially suppressed by an asymmetric mass cut around the nominal $B^{0}$ mass, $m_{K \pi \mu \mu} \in[5150,5700] \mathrm{MeV}$.

On average $12 \%$ of events in data had more than one reconstructed $B^{0}$ candidate passing all selection cuts. In the first step of best-candidate selection, the candidate with the smallest value of the $B^{0}$ vertex $\chi^{2} / N D F$ was retained. However, in about $96 \%$ of cases the multiple candidates arose from four-track combinations with swapped kaon and pion mass assignment. As these candidates have the same value of $B^{0}$ vertex $\chi^{2} / N D F$, the candidate with the smallest value of $\left|m_{K \pi}-m_{K^{*}}\right| / \sigma_{m_{K \pi}}$ was selected. Here $m_{K \pi}$ is mass of the reconstructed $K^{*}$ candidate, $\sigma_{m_{K \pi}}$ its uncertainty and $m_{K^{*}}$ is the world average value of $K^{*}$ mass [7].

After the selection, some of the $B^{0}$ candidates had an incorrect flavour tag (mistag). The mistag probability of $B^{0}\left(\overline{B^{0}}\right)$ was determined from signal MC to be $0.1088 \pm 0.0005(0.1086 \pm 0.0005)$. This leads to changes in the values of measured decay angles, namely in swap of their signs. The corresponding dilution of measured values of $S_{i}$ parameters was included as systematic uncertainty.

Events with $q^{2} \in[0.98,1.1] \mathrm{GeV}^{2}$ were vetoed in order to remove potential contamination from $\phi(1020)$. The remaining data with $q^{2} \in[0.04,6.0] \mathrm{GeV}^{2}$ were analysed. Two control samples containing $B^{0}$ decays to $J / \psi K^{*}$ and $\psi(2 S) K^{*}$ were defined in regions of $q^{2} \in[8,11] \mathrm{GeV}^{2}$ and $[12,15] \mathrm{GeV}^{2}$. As the background, including contributions from radiative decays of $J / \psi$, increases above $q^{2}$ of $6 \mathrm{GeV}^{2}$, data in this region were not used in the analysis.

In total 787 events were selected and analysed in $q^{2}$ bins $[0.04,2.0],[2.0,4.0]$ and $[4.0,6.0]$ 
$\mathrm{GeV}^{2}$. To allow comparison with results of other experiments and with theoretical predictions, additional fits were performed to wider bins of $q^{2} \in[0.04,4.0],[1.1,6.0]$ and $[0.04,6.0] \mathrm{GeV}^{2}$.

\section{Fitting procedure}

Extended unbinned maximum-likelihood fits of the signal decay were performed on the data for each $q^{2}$ bin. The likelihood is given by

$$
\mathscr{L}=\frac{\mathrm{e}^{-n^{\prime}}}{n !} \prod_{k=1}^{n} \sum_{l} n_{l} P_{k l}\left(m_{K \pi \mu \mu}, \cos \theta_{K}, \cos \theta_{L}, \phi ; \widehat{p}, \widehat{\theta}\right),
$$

where $n$ is the total number of events, the sum runs over signal and background components, $n_{l}$ is the fitted yield for the $l^{\text {th }}$ component, $n^{\prime}$ is the sum over $n_{l}$ and $P_{k l}$ is the probability density function (pdf) evaluated for $k^{\text {th }}$ event and $l^{\text {th }}$ component. The discriminating variables are $m_{K \pi \mu \mu}, \cos \theta_{K}$, $\cos \theta_{L}$ and $\phi$. The $\hat{p}$ are the parameters of interest $F_{\mathrm{L}}$ and $S_{i}$ and the $\widehat{\theta}$ the nuisance parameters.

The signal mass distribution was modelled by a Gaussian function with width scaled by the per-event uncertainty of the reconstructed mass. The mass distribution of combinatorial background was described by an exponential function and second-order Chebychev polynomials were used to model the angular distributions. The MC sample generated with uniform $\cos \theta_{K}, \cos \theta_{L}$ and $\phi$ distributions was used to study the signal reconstruction and acceptance. The acceptance function was defined as the ratio of reconstructed and generated distributions in $\cos \theta_{K}, \cos \theta_{L}$ and $\phi$. It was assumed to factorise for each angular distribution and it was described by sixth-order (second-order) polynomial for $\cos \theta_{K}$ and $\cos \theta_{L}(\phi)$.

A two-step fit was performed for each $q^{2}$ bin: in the first step the $B^{0}$ invariant mass distribution was fitted with mean values of the mass and the scale factor extracted from fits to data in the control regions. In the second step the $\cos \theta_{K}, \cos \theta_{L}$ and $\phi$ variables were included in the likelihood fit. The nuisance parameters, i.e. mean mass, scale factor, signal and background yields and the shape of the mass distribution of combinatorial background were fixed to the results of the first step.

\subsection{Backgrounds}

Several samples of background processes were studied in order to identify contributions to be included in the fit model. Backgrounds included inclusive samples $b \bar{b} \rightarrow \mu^{+} \mu^{-} X$ and $c \bar{c} \rightarrow$ $\mu^{+} \mu^{-} X$, as well as number of exclusive samples. Decays possibly contributing to the signal region were $\Lambda_{b} \rightarrow \Lambda(1520) \mu^{+} \mu^{-}, \Lambda_{b} \rightarrow p K^{-} \mu^{+} \mu^{-}, B^{+} \rightarrow K^{(*)+} \mu^{+} \mu^{-}$and $B_{s}^{0} \rightarrow \phi \mu^{+} \mu^{-}$. However, their contribution was small and thus they were not included in the fits and they were accounted for as systematic uncertainties.

A background peaking in $\cos \theta_{K}$ and $\cos \theta_{L}$ distributions not considered above was observed in data. A peak found around $\cos \theta_{K}=1$ comes from two sources: one of them is $B^{+} \rightarrow K^{+} \mu \mu$ or $B^{+} \rightarrow \pi^{+} \mu \mu$ decays with an additional track from the event, the second source are events with two charged tracks reconstructed as a $K^{*}$ candidate passing all cuts. Another peak was found in the region of $\cos \theta_{L}= \pm 0.7$ and was associated with partially reconstructed $B \rightarrow D \mu^{+} \mu^{-} X$ decays. MC simulated events containing $D^{0} \rightarrow K \pi, D^{+} \rightarrow K \pi \pi$ and $D_{s}^{+} \rightarrow K K \pi$ were found to accumulate in this region due to the kinematics of the decays. Both these backgrounds were treated as sources of systematic uncertainties and were not included in the fit. 


\subsection{Systematic uncertainties}

The treatment of systematic uncertainties of angular parameters was based on observed fit biases in modified pseudo-experiments or on comparison of nominal and modified fit. The most significant contributions were the following, in order of importance:

- Combinatorial $K \pi$ background peaking at $\cos \theta_{K}=1$ : uncertainty was computed comparing results of the nominal fit with that where data above $\cos \theta_{K}=0.9$ were excluded from fit.

- Background peaking around values of $\left|\cos \theta_{L}\right|=0.7$ : a veto was implemented to remove events, where after assigning two or three tracks mass hypotheses consistent with $D^{0} \rightarrow K \pi$, $D^{+} \rightarrow K \pi \pi$ and $D_{s}^{+} \rightarrow K K \pi$ decays, the reconstructed mass fell in a window of $30 \mathrm{MeV}$ around the $D^{0}, D^{+}$or $D_{s}^{+}$mass. A similar veto was implemented to reject $B^{+} \rightarrow K^{+}\left(\pi^{+}\right) \mu \mu$ candidates with mass less than $50 \mathrm{MeV}$ from $B^{+}$mass. The results of the fit with and without the veto were than compared.

- Background angular pdf shape: data were refitted using third-order Chebychev polynomials.

- Factorisation of acceptance function: uncertainty was computed as difference between the generated angular parameter values and results of fitting MC signal sample with acceptance function derived from it.

- Combinatorial background: nominal fit results were compared to those with a reduced mass range of $m_{K \pi \mu \mu} \in[5200,5700] \mathrm{MeV}$.

- Inner detector alignment and knowledge of magnetic field: track $p_{\mathrm{T}}$ in data sample was shifted according to uncertainties from biases in momentum scale.

- Intrinsic bias of the maximum-likelihood estimator: ensembles of MC simulated events were used to estimate the bias in the extracted values of parameters.

Lower uncertainties arise from other sources, such as difference of the $p_{\mathrm{T}}$ spectra of $B^{0}$ candidates in data and $\mathrm{MC}$ simulation, $\mathrm{S}$-wave contribution (candidates coming from non-resonant $B^{0} \rightarrow K \pi \mu^{+} \mu^{-}$decay), nuisance parameters, exclusive peaking backgrounds and dilution of measured parameters due to flavour mistag. However, the systematic uncertainties are smaller than the statistical ones for all measured parameters.

\section{Results}

The fitted event yields for all $q^{2}$ bins are summarised in Table 1. Figure 3 shows an example of fits to $q^{2}$ bin of $[0.04,2.0] \mathrm{GeV}^{2}$ for folding scheme $S_{4}$.

The distributions of $F_{\mathrm{L}}$ and $S_{i}$ parameters as a function of $q^{2}$ are shown in Figure 4. Distributions of $P_{i}$ parameters computed from $S_{i}$ using transformations (1.2) are shown in Figure 5. The figures also show results of theoretical predictions of SM by various groups: Ciuchini et al. (CFFMPSV) [8], Descotes-Genon et al. (DHMV) [9] and Jäger and Martin Camalich (JC) [10]. Experimental results from LHCb [11], CMS [12], Belle [13, 14] and BaBar [15] are also shown.

There is a good agreement between theoretical predictions and the ATLAS measurement. The exceptions are $P_{4}^{\prime}$ and $P_{5}^{\prime}$ measurements in $q^{2}=[4.0,6.0] \quad \mathrm{GeV}^{2}$ and $P_{8}^{\prime}$ in $q^{2}=[2.0,4.0] \mathrm{GeV}^{2}$. The observed deviation from the SM prediction in $P_{4}^{\prime}$ is approximately 2.5 standard deviations from the DHMV calculation. The deviation of $P_{5}^{\prime}$ is consistent with the deviation reported by the LHCb Collaboration [11] and is 2.7 standard deviations from the DHMV calculation, with lower 


\begin{tabular}{r|cc}
\hline \hline$q^{2}\left[\mathrm{GeV}^{2}\right]$ & $n_{\text {sig }}$ & $n_{\text {bck }}$ \\
\hline$[0.04,2.0]$ & $128 \pm 22$ & $122 \pm 22$ \\
{$[2.0,4.0]$} & $106 \pm 23$ & $113 \pm 23$ \\
{$[4.0,6.0]$} & $114 \pm 24$ & $204 \pm 26$ \\
\hline$[0.04,4.0]$ & $236 \pm 31$ & $233 \pm 32$ \\
{$[1.1,6.0]$} & $275 \pm 35$ & $363 \pm 36$ \\
{$[0.04,6.0]$} & $342 \pm 39$ & $445 \pm 40$ \\
\hline \hline
\end{tabular}

Table 1: The values of fitted signal and background yields $n_{\text {sig }}$ and $n_{\mathrm{bkg}}$, uncertainties are statistical only [2].
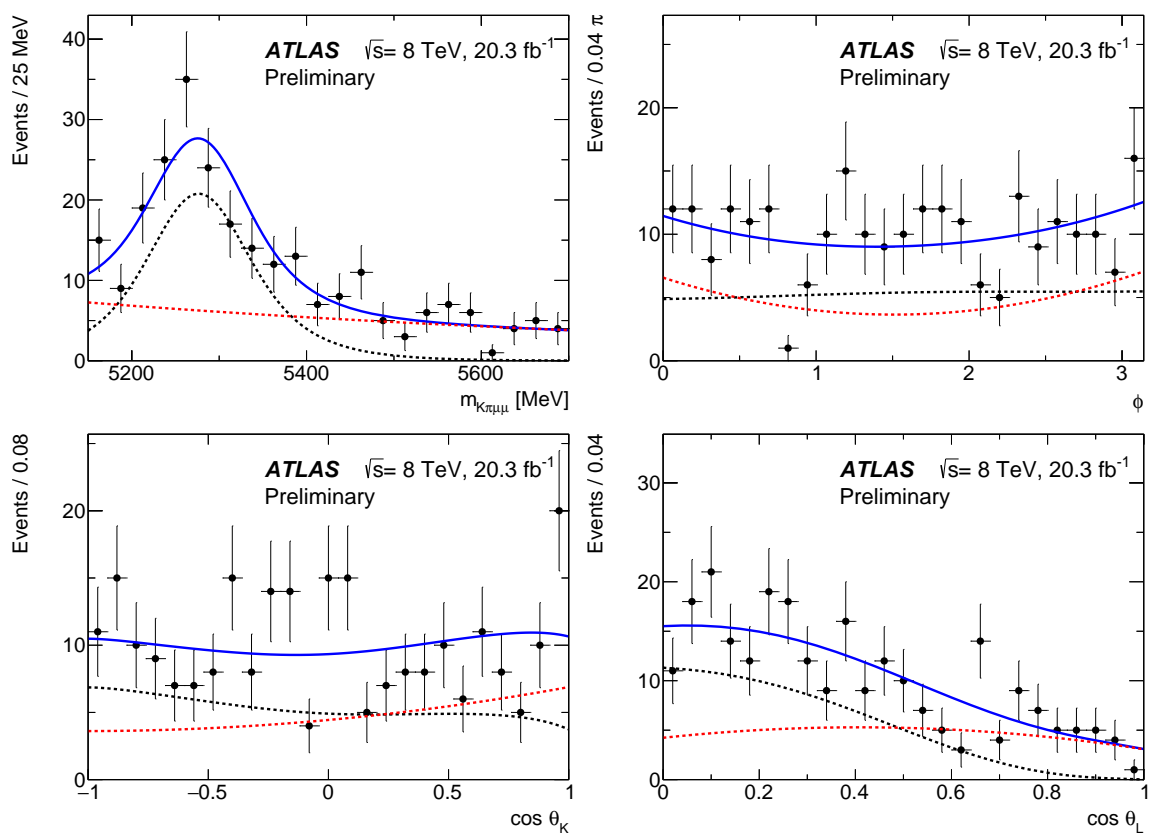

Figure 3: The fitted distributions of $m_{K \pi \mu \mu}, \cos \theta_{K}, \cos \theta_{K}$ and $\phi$ obtained for $q^{2} \in[0.04,2.0]$ in $S_{4}$ folding scheme [2]. The data uncertainties are statistical only. The blue solid line is a projection of the total pdf and the black (red) dashed lines represent the signal (background) components.

significance for other calculations. However, all measurements are within three standard deviations from quoted predictions and after including experimental and theoretical uncertainties are found in agreement with the SM contributions to this decay.

\section{Conclusions}

The angular analysis of rare decay $B_{d}^{0} \rightarrow K^{*} \mu^{+} \mu^{-}$was presented using data collected by the ATLAS experiment collected in 2012 at centre-of-mass energy of $\sqrt{s}=8 \mathrm{TeV}$ with integrated luminosity of $20.3 \mathrm{fb}^{-1}$. Angular parameters $F_{\mathrm{L}}, S_{i}$ and $P_{i}$ were extracted from the data using an extended unbinned maximum-likelihood fit in six bins of $q^{2}$, up to $6 \mathrm{GeV}^{2}$. All results were found to be within three standard deviation from theoretical predictions of the Standard Model. 

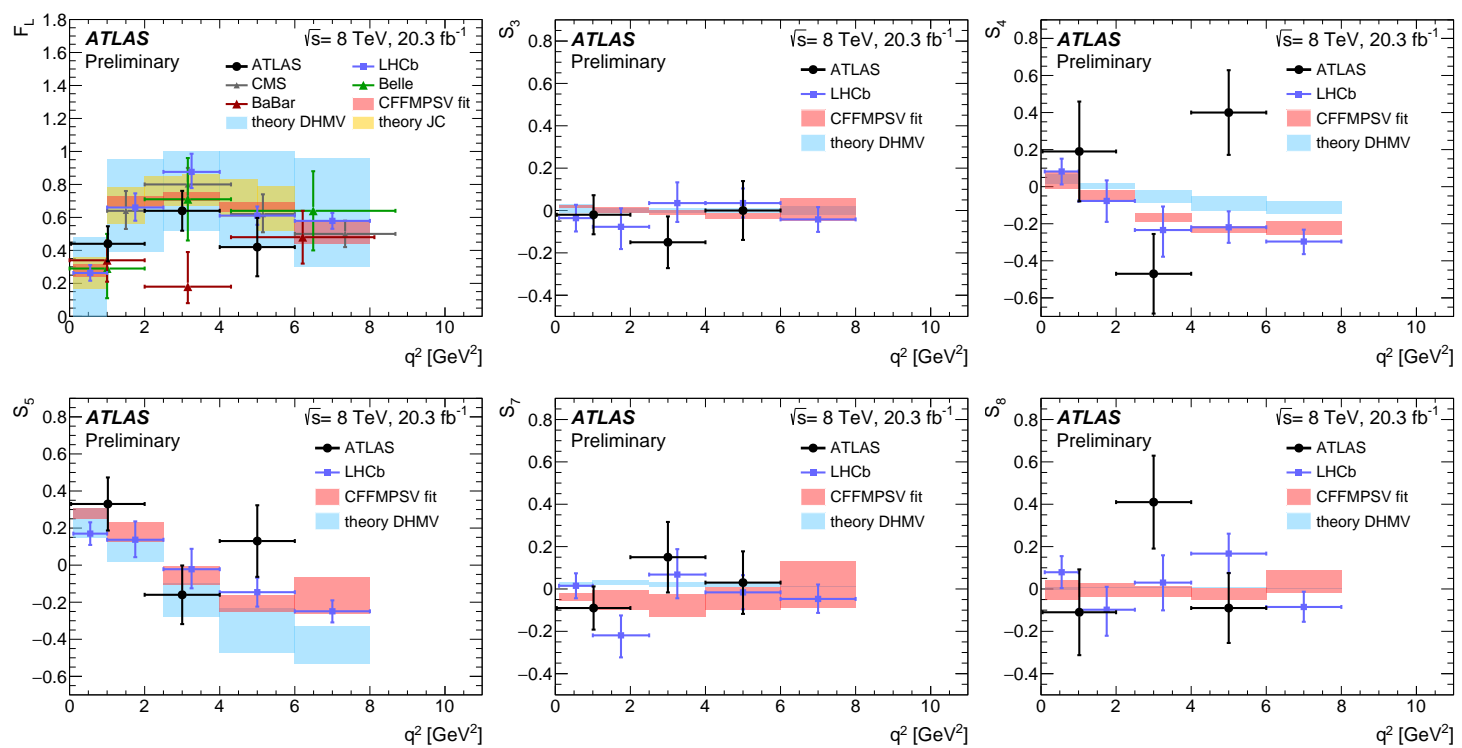

Figure 4: The measured values of $F_{\mathrm{L}}, S_{3}, S_{4}, S_{5}, S_{7}$ and $S_{8}$ parameters compared with predictions from theoretical groups and results from other experiments as discussed in the text [2]. Total uncertainties are plotted for all measurements and theoretical predictions.
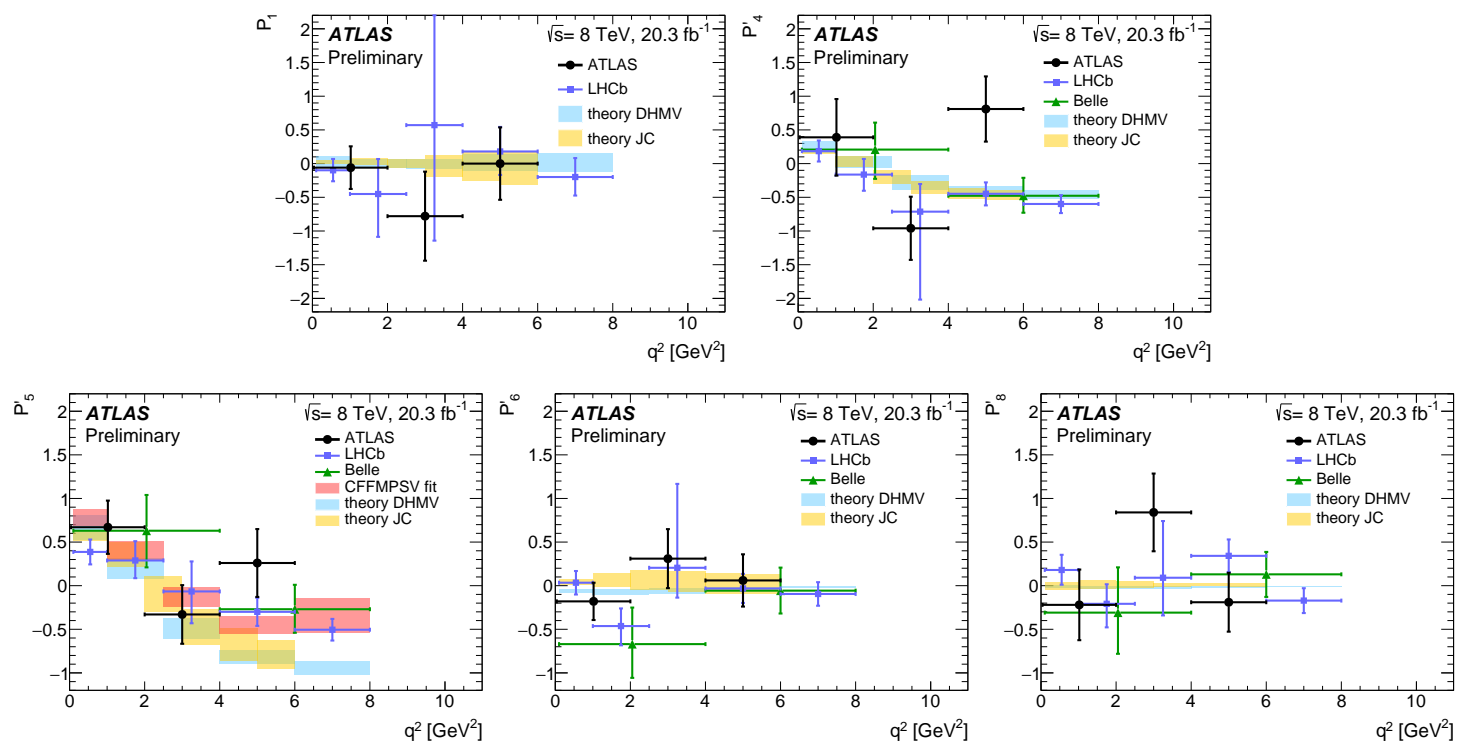

Figure 5: The measured values of $P_{1}, P_{4}^{\prime}, P_{5}^{\prime}, P_{6}^{\prime}$ and $P_{8}^{\prime}$ parameters compared with predictions from theoretical groups and results from other experiments as discussed in the text [2]. Total uncertainties are plotted for all measurements and theoretical predictions. 


\section{References}

[1] L. Evans and P. Bryant, LHC Machine, JINST 3 (2008) S08001.

[2] ATLAS Collaboration, Angular analysis of $B_{d}^{0} \rightarrow K^{*} \mu^{+} \mu^{-}$decays in pp collisions at $\sqrt{s}=8 \mathrm{TeV}$ with the ATLAS detector, ATLAS-CONF-2017-023, https://cds.cern.ch/record/2258146.

[3] LHCb Collaboration, R. Aaij et al., Differential branching fraction and angular analysis of the decay $B^{0} \rightarrow K^{* 0} \mu^{+} \mu^{-}$, JHEP 08 (2013) 131, arXiv:1304. 6325 [hep-ex].

[4] LHCb Collaboration, R. Aaij et al., Measurement of Form-Factor-Independent Observables in the Decay $B^{0} \rightarrow K^{* 0} \mu^{+} \mu^{-}$, Phys. Rev. Lett. 111 (2013) 191801, arXiv:1308.1707 [hep-ex].

[5] S. Descotes-Genon, T. Hurth, J. Matias and J. Virto, Optimizing the basis of $B \rightarrow K^{*} \ell^{+} \ell^{-}$observables in the full kinematic range, JHEP 05 (2013) 137, arXiv:1303. 5794 [hep-ph].

[6] ATLAS Collaboration, The ATLAS Experiment at the CERN Large Hadron Collider, JINST 3 (2008) S08003.

[7] K. A. Olive et al., The Review of Particle Physics, Chin. Phys. C 38 (2014) 090001.

[8] M. Ciuchini, M. Fedele, E. Franco, S. Mishima, A. Paul, L. Silvestrini et al., $B \rightarrow K^{*} \ell^{+} \ell^{-}$decays at large recoil in the Standard Model: a theoretical reappraisal, JHEP 06 (2016) 116, arXiv:1512.07157 [hep-ph].

[9] S. Descotes-Genon, L. Hofer, J. Matias and J. Virto, On the impact of power corrections in the prediction of $B \rightarrow K^{*} \mu^{+} \mu^{-}$observables, JHEP 12 (2014) 125, arXiv:1407. 8526 [hep-ph].

[10] S. Jäger and J. Martin Camalich, Reassessing the discovery potential of the $B \rightarrow K^{*} \ell^{+} \ell^{-}$decays in the large-recoil region: SM challenges and BSM opportunities, Phys. Rev. D 93 (2016) 014028 , arXiv:1412.3183 [hep-ph].

[11] LHCb Collaboration, R. Aaij et al., Angular analysis of the $B^{0} \rightarrow K^{* 0} \mu^{+} \mu^{-}$decay using $3 f b^{-1}$ of integrated luminosity, JHEP 02 (2016) 104, arXiv:1512. 04442 [hep-ex].

[12] CMS Collaboration, Measurement of the $P_{1}$ and $P_{5}^{\prime}$ angular parameters of the decay $B^{0} \rightarrow K^{* 0} \mu^{+} \mu^{-}$ in proton-proton collisions at $\sqrt{s}=8 \mathrm{TeV}, \mathrm{CMS}-\mathrm{PAS}-\mathrm{BPH}-15-008$, https://inspirehep.net/record/1518992.

[13] Belle Collaboration, J.-T. Wei et al., Measurement of the Differential Branching Fraction and Forward-Backward Asymmetry for $B \rightarrow K^{(*)} l^{+} l^{-}$, Phys. Rev. Lett. 103 (2009) 171801, arXiv:0904.0770 [hep-ex].

[14] Belle Collaboration, S. Wehle et al., Lepton-Flavor-Dependent Angular Analysis of $B \rightarrow K^{*} \ell^{+} \ell^{-}$, Phys. Rev. Lett. 118 (2017) 111801, arXiv:1612.05014 [hep-ex].

[15] BaBar Collaboration, J. P. Lees et al., Measurement of angular asymmetries in the decays $B \rightarrow K^{*} \ell^{+} \ell^{-}$, Phys. Rev. D 93 (2016) 052015, arXiv:1508.07960 [hep-ex]. 